Revue d'histoire de l'Amérique française

REVUE D.HISTOIRE DE L'AMÉRIQUE FRANÇAISE

\title{
Le Nouveau Monde et la question du Nord-Ouest
}

\section{Léon Pouliot}

Volume 11, numéro 3, décembre 1957

URI : https://id.erudit.org/iderudit/301846ar

DOI : https://doi.org/10.7202/301846ar

Aller au sommaire du numéro

Éditeur(s)

Institut d'histoire de l'Amérique française

ISSN

0035-2357 (imprimé)

1492-1383 (numérique)

Découvrir la revue

Citer cet article

Pouliot, L. (1957). Le Nouveau Monde et la question du Nord-Ouest. Revue

d'histoire de l'Amérique française, 11(3), 353-360.

https://doi.org/10.7202/301846ar d'utilisation que vous pouvez consulter en ligne.

https://apropos.erudit.org/fr/usagers/politique-dutilisation/ 


\section{LE « NOUVEAU MONDE » ET LA QUESTION DU NORD-OUEST}

Quand le gouvernement canadien décida d'unir à la Confédération les vastes territoires du Nord-Ouest, la cause des Métis n'eut pas de plus zélé défenseur que le Nouveau Monde, journal politique et religieux, dont le numéro-prospectus paraissait à Montréal, le 10 août 1867.

Si la majorité des actionnaires du Nouveau Monde se recrutait dans le clergé et si le secrétaire du journal, l'abbé Godefroy Lamarche, résidait à l'évêché, la rédaction était laïque. Parmi les rédacteurs les plus connus qui se sont succédé de 1867 à 1878, il suffira de nommer ici l'avocat Joseph Royal, plus tard lieutenant-gouverneur du Manitoba, l'avocat Édouard Lefebvre de Bellefeuille, auteur de plusieurs études juridiques et l'avocat Alphonse Desjardins, qui allait devenir député d'Hochelaga, sénateur et ministre à Ottawa. C'est Royal qui est rédacteur-en-chef en 1869-1870.

Entre plusieurs articles du Nouveau Monde sur la question du Nord-Ouest, nous publions celui du 27 avril 1870, parce qu'il nous paraît exprimer et la politique du journal sur le sujet et l'opinion publique du Bas-Canada. Mais il ne sera pas inutile de le replacer dans son contexte historique. A l'automne de 1869, l'honorable William McDougall est nommé lieutenant-gouverneur de la Rivière-Rouge et il se met en route pour occuper son poste. Mais, pour des raisons que nous n'avons pas à raconter ici, il ne peut entrer dans le pays et doit revenir à Ottawa tout penaud et aigri.

Pendant ce temps, le Nord-Ouest se donne un gouvernement provisoire, présidé par Louis Riel. Au début de 1870, exécution de Scott, jugée nécessaire par le gouvernement provisoire. Cet acte est l'occasion de violentes assemblées de protestation (indignation meetings) dans les principales villes du Haut-Canada; exemple suivi par les Canadiens de langue anglaise de Montréal. 
Le gouvernement paraît débordé, incapable de dominer la situation. Il invite des délégués du Nord-Ouest pour trouver une solution au problème; mais en même temps, il décrète l'envoi à la Rivière-Rouge d'une expédition militaire. Il ne peut pas ou il n'ose pas empêcher l'arrestation par la police du Haut-Canada de ses invités à Ottawa: les délégués du Nord-Ouest.

A travers tout cela, se lève cependant une lueur d'espoir: le ministère, tout à coup, semble venir à de meilleurs sentiments et préférer les moyens pacifiques aux moyens violents. C'est alors, 27 avril 1870, que paraît l'article éditorial du Nouveau Monde qu'on lira, sans doute, avec intérêt et plaisir.

\section{L'HORIZON S'ÉCLAIRCIT}

Depuis le jour où les chefs de la Confédération canadienne convinrent de la nécessité d'ajouter aux provinces de la vallée du Saint-Laurent et du Golfe le vaste territoire du Nord-Ouest, besoin politique et nécessité nationale, dont nous n'avons jamais cessé nous-même d'être convaincu, la ligne de conduite du Nouveau Monde fut toute tracée. Les circonstances avaient voulu que nous fussions de suite au courant de la question; nous fîmes nos efforts pour que l'opinion publique s'en occupât et comprît toute la gravité des intérêts qui se trouvaient mis en jeu. Correspondances de la Rivière-Rouge, informations particulières toutes aussi authentiques, documents officiels, étude sur chaque fait à mesure qu'il se produisait, rien ne fut négligé par nous pour jeter le plus de lumière possible sur cette question avant que sa solution ne fût décidée par nos ministres, soit dans un sens soit dans l'autre. ${ }^{1}$

1 On a prétendu que Riel était l'auteur des lettres de la Rivière-Rouge publiées dans le Nouveau Monde, fin de 1868 et début de 1869. A notre avis, une seule pourrait lui être attribuée, celle du 23 février 1869, signée L.R. et dont l'auteur se donne comme Métis. Les autres lettres sont signées $X$ ou $\mathrm{X}$ Y Z., et leur contenu, selon nous, ne permet pas de les attribuer à Louis Riel plutôt qu'à un autre. - Le 30 octobre 1868, le Nouveau Monde analysait le rapport de Dawson sur la Rivière-Rouge. - L'Esquisse sur le NordOuest américain par Mgr Taché parut dans le Nouveau Monde (25 août-14 octobre 1869) avant de paraître en forme de volume, au printemps de 1870 . Voici comment l'Esquisse était présentée aux lecteurs: 
Qui connaissait le Nord-Ouest avant 1868 ? Personne ou à peu près. Il est bien vrai que dans les longues veilles d'hiver, les légendes racontées par nos anciens sur leurs exploits dans ces lointaines contrées ont vivement ému l'enfance de chacun de nous. En ce temps-là, les voyageurs de là-haut partaient de Lachine en canot, remontaient l'Outaouais, descendaient dans le lac Huron par la Rivière des Français, et traversaient le lac Supérieur dans toute sa longueur pour ensuite commencer la route si difficile, si longue et si périlleuse qui menait de la Baie du Tonnerre aux comptoirs de la Rivière-Rouge.

Dans ces expéditions pacifiques du commerce ou du missionnaire catholique, nos gens portaient le plumet. Les plus durs à la fatigue, ils étaient encore les plus solides sous la bricole et les plus braves dans le danger. C'est qu'aussi la Compagnie du NordOuest n'engageait à son service que les plus beaux et les plus capables de nos gars.

Presque chaque famille comptait alors dans les pays d'en haut un des siens qui n'était jamais revenu, et dont le vieux père, en racontant l'histoire, disait, en branlant douloureusement la tête, qu'il n'avait jamais eu depuis ni vent ni nouvelle. Beaucoup, en effet, de ces brillants et vigoureux jeunes gens ont péri dans les cataractes des rivières, dans les tempêtes des grands lacs ou sous la balle du Sauvage; mais il en a survécu de ceux qui ne sont jamais revenus, et ceux-là ont formé le noyau de cette population de métis, dont on a tant parlé depuis huit mois. Race forte, énergique, patiente,

«Le Nouveau Monde doit le déclarer: il ne recherche, dans cette publication, dont l'honore un des premiers prélats canadiens, ni la facile satisfaction de son amour-propre, ni la preuve d'une thèse politique quelconque (.....) Il s'agit ici de sauvegarder les intérêts d'une population et d'un pays contre l'ambition, l'avidité et le fanatisme; et plus on connaîtra la vérité sur cette population et ce pays, mieux on en connaîtra les besoins et les destinées. L'annexion du Nord-Ouest fut une nécessité d'Etat, en même temps qu'une sage mesure politique, nous l'avons déjà dit; mais pourquoi s'illusionner sur les seuls avantages de cette annexion au point de vue matériel ? N'est-ce pas s'exposer à de graves mécomptes? 》- Le Nouveau Monde tient ses lecteurs au courant des événements; il reproduit les nouvelles vraies ou fausses communiquées aux journaux par les agences télégraphiques; il publie au texte les principaux articles de la New Nation, journal du gouvernement provisoire, du Nor'Wester; il discute les opinions émises dans les journaux de langue anglaise, etc., etc. 
soumise, morale, religieuse, elle rappelle les Acadiens d'autrefois: tels sont les colons de la RivièreRouge. Nous avons publié l'histoire généalogique de plusieurs de ces familles, et nous devions continuer quand les événements nous ont forcés de remettre à plus tard la suite de ces détails intéressants. ${ }^{2}$

Outre les légendes de cette ultima Thule, ${ }^{3}$ nous connaissons encore un peu le Nord-Ouest, mais en Bas-Canada seulement, par les relations de missions publiées de temps à autre dans les Annales de la Propagation de la Foi. Quelquefois, un missionnaire de passage ici faisait un sermon sur les progrès du christianisme dans ces lointains pays; on parlait du départ de quelques-unes de nos héroïques Sœurs de charité, qui allaient s'y vouer à l'instruction des enfants; de nos jeunes prêtres partaient d'autres fois avec le saint et illustre évêoue Taché : voilà tout ce qu'on savait des territoires du Nord-Ouest avant 1868. C'était une terre de chasse pour l'avide facteur de la Cie de la Baie d'Hudson, et un territoire de missions pour le prêtre catholique et la Sceur de charité; pour l'enfance, c'était par excellence le pays peuplé de Canadiens légendaires.

Le Ministère en savait-il davantage? Non. Egaré, mal renseigné par les rapports de MM. Hind et Dawson, ${ }^{4}$ il croyait que le Nord-Ouest était la terre

231 octobre 1868: «Notes sur quelques habitants de la Rivière-Rouge »: Jean-Baptiste Charbonneau, Pierre Thibert, Toussaint Lussier, Charles Laurance, Jean-Baptiste Lapointe dit Désautels; 10 février 186.9: "Notice sur quelques Canadiens établis à la Rivière-Rouge »: Michel Dumas, François Larivière, Paul Proulx, Jean-Marie Boucher, Emmanuel Beaugrand (dit Champagne), André Gaudry, Joseph Vandal, Jean-Baptiste Desrosiers, Augustin Racette; 29 mars 1869: «Recensement de quelques familles canadiennes à la Rivière-Rouge »: François Boudreau de Graveline, Jean-Baptiste Valiquette, Antoine Dupuis, Joseph Touron, Théophile Jetté; 16 avril 1869: «Notes sur quelques Canadiens établis à la Rivière-Rouge »: Dominique Ducharme, Onésime Manseau. - Ces notes ne sont pas signées. Il est manifeste qu'elles ont été rédigées sur les lieux. Par qui? Nous ne le savons pas avec certitude; mais nous pensons tout naturellement à l'abbé Georges Dugas.

3 Thulé: «nom donné par les Romains à une île du nord de l'Europe (probablement une des Shetland), qui marquait l'extrême limite N. du monde ». Dict. Larousse.

${ }^{4}$ Henry Youle Hind (1823-1908). Né en Angleterre, arrive au Canada en 1846; professeur de chimie, de mathématiques et de géologie à l'Ecole Normale et à l'Université de Toronto. Attaché comme géologue à l'expédition de la Rivière-Rouge, décrétée par le gouvernement canadien en 1857. 
promise de l'avenir et que la fertilité des prairies de la Rivière-Rouge, de l'Assiniboine, de la Kisiskatchewan égalait au moins celle des grands plateaux situés au sud des lacs Erié et Michigan !... De la population, il n'en savait pas un mot; de fait, elle n'avait pas d'existence politique; seule la Compagnie de la Baie d'Hudson possédait, gouvernait et régnait.

Cette double ignorance du gouvernement canadien a été le secret de toutes ses fautes et de tous nos malheurs avec le Nord-Ouest.

Il y a deux ans que, sous l'impulsion d'une cause religieuse et nationale à défendre, le Nouveau Monde a fait de la question du Nord-Ouest l'un des principaux objets de sa sollicitude.

Répandre des notions exactes sur la condition sociale de la colonie de la Rivière-Rouge, - corriger les grossières exagérations qui se sont débitées et qui se débitent encore sur le sol et sur le climat de ces territoires - , conseiller, dans tous les cas, une politique de prudence, de justice, de ménagements et de paix avec la population du pays: telle a été notre ligne de conduite invariable. Et aujourd'hui, nous pouvons dire que si les voix qui ont parlé dans nos colonnes, maintes et maintes fois, eussent été écoutées et respectées, il y a six mois que la question du Nord-Ouest ne chargerait plus l'horizon politique de la tempête qui nous menace. Au lieu de cela, les ministres se voient, en ce moment, obligés de finir par où ils auraient dû commencer.

Nous l'avons déjà dit, et nous le répétons pour l'enseignement de l'avenir: nos ministres, dans cette question, ont été débordés, devancés et menés dès l'origine par une province ambitieuse et résolue, le Haut-Canada.

Narrative of the Canadian Red River exploring expedition of 1857, and of the Assiniboine and Saskatchewan exploring expedition of 1858. (2 vol., Toronto, 1859, Londres, 1860 ).

Simon James Dawson (1820-1902). Chargé par le gouvernement canadien, en 1857, d'explorer la région qui s'étend du lac Supérieur à la Saskatchewan. Son rapport (1859) fut l'un des premiers à attirer l'attention sur la possibilité de coloniser les territoires du Nord-Ouest. En 1868, on lui demande d'indiquer une voie de communication avec la Rivière-Rouge. Et son rapport est inséré dans les documents officiels du Canada. Report on the line of route between Lake Superior and the Red River Settlement (Ottawa, 1868). 
C'est le Haut-Canada qui a demandé les explorations de Hind et de Dawson; c'est le Haut-Canada qui a surtout poussé la question dans les Chambres; c'est le Haut-Canada qui seul a fourni les fonctionnaires que le gouvernement canadien a eu le malheur d'envoyer d'abord à la Rivière-Rouge ; ${ }^{5}$ c'est le HautCanada qui a fourni le premier Lieutenant-Gouverneur qui devait y organiser le régime politique; et après que tant de fautes ont été commises, c'est encore le Haut-Canada qui nous fait violer le droit des gens d'une manière encore inouie dans l'histoire des peuples civilisés ; ${ }^{6}$ c'est encore le Haut-Canada qui force la main au Cabinet pour organiser contre les Métis une expédition militaire.

Certes, en présence d'un tel entêtement à subir l'influence d'une politique locale, tyrannique, égoïste et injuste; en face du mépris que l'on a fait des avis et des bons conseils qui indiquaient où devait aboutir une telle conduite; en présence des dénigrements qui accueillaient dans les régions semi-officielles ses paroles de vérité et de paix, le Nouveau Monde a usé librement de son franc-parler. Nous avons élevé la voix sans passion, mais avec l'accent d'une conviction inébranlable et l'émotion qu'inspire le spectacle de l'injustice.

L'opinion publique a été avec nous et comme aujourd'hui les nouvelles d'Ottawa sont à la paix, nous nous en réjouissons et formons le vœu que de telles espérances reçoivent enfin leur pleine et entière réalisation. Ce sera, certes, une grande victoire que l'attitude ferme, calme, énergique et froidement déterminée de l'opinion publique du Bas-Canada aura remportée sur le fanatisme ambitieux et bigot, s'il est vrai que le gouvernement se dispose à régler la question par une politique de conciliation, de paix et de justice. Cependant, avant que d'approuver cette paix annoncée hier soir aux Chambres par le Premier

5 Il faudrait pourtant mentionner J.-A.-N. Provencher, rédacteur de la Minerve, qui accompagna McDougall à la Rivière-Rouge, en qualité de secrétaire. Minime concession accordée par le ministère, à la dernière heure.

6 Alllusion à l'arrestation par la police du Haut-Canada des trois délégués du Nord-Ouest venus pour conférer avec le gouvernement canadien, à l'invitation de celui-ci. 
Ministre, nous voulons bien la connaître et juger jusqu'à quel point elle est digne, équitable et patriotique. N'ayant point ménagé au ministère notre censure sur une politique qui s'annonçait par mille signes extérieurs, nous ne lui ménagerons pas non plus notre appui, s'il se déclare enfin pour un principe que nous n'avons cessé de défendre et d'affirmer. Let us have peace!

On aura remarqué que, dans ce long article, le nom de Riel n'est pas une seule fois prononcé. Et ce n'est pas, pour le Nouveau Monde tactique opportuniste, mais politique raisonnée. Tandis que le Haut-Canada ne concentre son attention que sur Riel, Riel le meurtrier de Scott, et dont on demande la tête à cor et à cri, le Nouveau Monde, qui nous paraît faire écho à l'opinion publique du Bas-Canada, a une vue plus large des choses; il ne confond pas la personne de Riel, président du gouvernement provisoire, avec la cause des Métis. Il condamne, lui aussi, l'exécution de Scott comme un acte inutile et souverainement nuisible au bien de la paix. Mais il ne se croit pas permis pour autant de s'ériger en juge de Riel et du gouvernement provisoire. Une erreur d'un chef dans un moment difficile n'entraîne pas condamnation de la cause qu'il défend, si, par ailleurs cette cause est juste.

Le Nouveau Monde va plus loin encore. Il refuse d'identifier la cause de Riel à celle de la Rivière-Rouge. Le Globe de Toronto affirmant que Riel est mécontent du bill du Manitoba, parce qu'il ne comporte pas l'amnistie générale, le Nouveau Monde répond, le 4 juin 1870, que cette amnistie relève du gouvernement impérial, non du gouvernement canadien. Et il ajoute:

M. Riel n'a dû son élévation à la tête des affaires de la Rivière-Rouge qu'en s'identifiant avec les intérêts de tous; le jour où son intérêt personnel s'en éloignera, nous osons dire qu'il sera brisé comme un roseau inutile. Si cependant, malgré les sages avis qui lui seraient donnés, M. Riel persistait, malgré tout, à empêcher l'entrée des troupes, ce n'est pas la colonie qui agirait, mais lui seul, en son propre et privé nom et à ses risques et périls. 
Et comme, en certains milieux, on continuait de voir dans le Nouveau Monde le défenseur per fas et nefas de Riel, il affirme clairement son attitude, le 7 juin:

L'Evénement nous permettra de lui faire observer que nous nous sommes toujours abstenus de parler de Riel personnellement dans tout ce que nous avons écrit depuis deux ans sur les affaires du NordOuest; c'est une régle que nous nous sommes tracée, dès les commencements et de laquelle nous avons peu dévié.

Notre confrère fait donc erreur en nous donnant à ses lecteurs comme «le plus zélé défenseur du Président du gouvernement provisoire du NordOuest ». Nous avons tâché, dans cette grave question, de dégager les événements et les principes de leurs auteurs, d'autant que la presse d'Ontario, adoptant la tactique contraire, s'est constamment efforcée de tout dénaturer, en s'attaquant davantage aux individus.

Cette attitude du Nouveau Monde nous paraît belle et méritoire. Il ne fait pas de doute qu'on y avait des sympathies pour la personne de Riel. Mais ce que l'on soutient avant tout, c'est une cause qui paraît juste; et parce que cette cause paraît juste, il faudrait la soutenir, même contre le chef du gouvernement provisoire, si celui-ci en venait à préférer son bien particulier au bien général.

De même, à cette époque - il en ira autrement plus tard le Nouveau Monde ne cache pas son admiration pour sir GeorgesÉtienne Cartier; il le cite en modèle aux députés du Bas-Canada; bien plus, il le compare à La Fontaine et ce n'est pas peu dire en un temps où ce dernier est considéré comme un véritable sauveur de la Patrie. Et cependant, cette estime de Cartier n'empêche pas le journal de dire franchement ce qu'il pense d'une politique dont Cartier est solidaire et dont il a sa large part de responsabilité. Sur ce point, Le Nouveau Monde a été fidèle à son programme de défendre les bons principes sans se lier, les yeux fermés, à un parti et de ne jamais défendre les individus aux dépens de la justice et de la vérité.

LÉON POULIOT, s.j. 